\title{
Satisfação da equipe de enfermagem com a imagem corporal
}

\section{Nursing team satisfaction with body image}

\author{
Jéssica Pereira Trentino ${ }^{1}$ Ana Carolina Cavalheiro ${ }^{2}$ Adriana Pereira da Silva Grilo ${ }^{2}$ André Oliveira Paggiaro $^{3}$ • \\ Ana Cláudia Puggina ${ }^{4}$
}

\section{RESUMO}

Objetiva-se identificar a satisfação dos profissionais da equipe de enfermagem com a imagem corporal, e avaliar se as características pessoais, profissionais e a necessidade do uso de adornos durante a jornada de trabalho interferem na satisfação com a imagem corporal. Estudo transversal quantitativo realizado com enfermeiros, técnicos e auxiliares de enfermagem por meio da Escala de Avaliação da Satisfação com a Imagem Corporal. A amostra foi de 182 profissionais, sendo $92,3 \%$ ( $n=168)$ mulheres, a idade média foi 32,74 anos $( \pm 8,3)$. 0 escore médio total foi de $83,8( \pm 14,1)$. Houve diferenças estatisticamente significativas na comparação dos escores na subescala "Preocupação com o Peso" com as variáveis sexo $(p=0,03)$ e estado civil ( $p=0,02)$. Concluí-se que a equipe de enfermagem referiu estar, em geral, moderadamente satisfeita com a imagem corporal. A preocupação com o peso foi maior nos indivíduos solteiros e do sexo masculino.

Palavras-chave: Imagem Corporal; Autoimagem; Cuidados de Enfermagem; Enfermagem.

\begin{abstract}
The aim is to identify the satisfaction of the nursing staff professionals with their body image and to assess whether the personal, professional characteristics and need to use adornments during working hours interfere with satisfaction with body image. A cross-sectional quantitative study carried out with nurses, technicians and nursing assistants using the Body Image Satisfaction Scale. The study was composed of 182 professionals, $92.3 \%$ $(n=168)$ female, with an average age of 32.74 years $( \pm 8.3)$. The average total score was $83.8( \pm 14.1)$. There were statistically significant differences when comparing the scores on the subscale "Worry with Weight" with the gender $(p=0.03)$ and marital status $(p=0.02)$. It concludes that nursing staff reported being moderately satisfied with their body image in general. The worry with weight was higher between the singles and males.
\end{abstract}

Keywords: Body Image; Self Concept; Nursing Care; Nursing. 


\section{INTRODUÇÃO}

A autoimagem corporal é parte essencial do mecanismo de identidade pessoal, uma vez que pode descrever muito sobre a pessoa, dando sinais de como pode ser seu comportamento e suas atitudes perante diferentes situações $^{1-2}$. É conceitualmente multidimensional, e suas representações são compostas tanto pela aparência, pelas medidas, pelos contornos e pela forma do próprio corpo, como também pelas respostas emocionais, sociais, culturais e satisfação em relação a essas percepções ${ }^{1-3}$. Consiste no que as pessoas pensam delas mesmas, e parte importante dela é a imagem corporal. A forma como elas se veem ou acreditam ser determina a maneira de falar, a postura corporal e o modo de se inter-relacionar com o outro ${ }^{4}$.

A sociedade e a cultura se relacionam com a imagem corporal na medida em que influenciam as preferências dos indivíduos e estabelecem o ideal de corpos perfeitos. Existe uma poderosa influência dos meios de comunicação nos padrões de beleza. A indústria da moda, a publicidade e a televisão, atualmente, tendem a impor um padrão mais magro e sinuoso, com enfoque no peso e na forma corporal. A insatisfação com a autoimagem pode estar associada ao estigma negativo atribuído aos que não se encaixam nesses padrões ${ }^{5}$.

Os padrões culturais foram modificaram no decorrer da história. Por exemplo, no século XIX e no início do século $\mathrm{XX}$, a gordura corporal era vista como símbolo de saúde e riqueza, e era valorizada e representada nas artes. Nos dias de hoje, há uma valorização da magreza como sinônimo de boa saúde, e a gordura passou a ser vista como sinal de doença ou falta de saúde ${ }^{5 .}$

Um dos desafios na estruturação do indivíduo é o caminho que segue para o desenvolvimento de sua imagem e o conhecimento do próprio corpo. Muitos problemas derivam da falta de conhecimento da natureza e da potencialidade que o corpo deveria proporcionar ${ }^{6}$.

A cultura ocidental moderna enfatiza a magreza, denigre o excesso de peso e estigmatiza indivíduos obesos, fazendo com que pessoas obesas internalizem essas mensagens, sendo crescente a insatisfação das pessoas com a própria aparência. Há constatações de que a obesidade está associada com a má imagem corporal, mas nem todas as pessoas obesas sofrem com esse problema ou são igualmente vulneráveis ${ }^{7}$.

Associado a isso, o estilo de vida da maioria da população, alicerçado nos avanços tecnológicos, contribui para a diminuição dos níveis de atividades físicas laborais e de lazer, fenômeno associado ao aumento do consumo de alimentos hipercalóricos e aos padrões de beleza que exigem perfis antropométricos cada vez mais magros ${ }^{8}$.

A imagem corporal pode ser entendida como uma forma de comunicação não verbal, ou seja, uma comunicação visual, já que, por meio da imagem, transmite-se uma mensagem que é captada e decodificada por quem a recebe.
Conhecer essa linguagem é importante não apenas por trazer informações sobre as pessoas, mas também porque o corpo físico é um centro intrínseco de informações de cada indivíduo ${ }^{4}$. Considerando a comunicação como um processo bidirecional, enquanto o profissional da enfermagem decodifica o paciente, o paciente também decodifica tudo em sua volta, incluindo a percepção de quem presta os cuidados, construindo conceitos e relações.

Aparência corporal, bem-estar físico e adequação de adornos e vestimentas ao contexto são fatores importantes em situações sociais e podem influenciar diretamente nas relações interpessoais. Os indivíduos buscam cada vez mais a tal imagem "perfeita", na tentativa de melhorar a autoimagem e a autopercepção, de forma a aprimorar, até mesmo, suas relações sociais ${ }^{9}$. A satisfação com a autoimagem e a imagem corporal diminui a sensação de insegurança e ansiedade, tendo em vista que, quando o profissional se sente feliz com sua aparência, torna-se mais seguro para se relacionar com os pacientes e outros membros da equipe de trabalho4.

Ao investigar características de personalidade e imagem corporal de mulheres que buscam cirurgia estética, constatou-se que quanto maior a insatisfação com a própria aparência corporal, mais elevada a atitude defensiva e maior a sensibilidade afetiva. As mulheres investigadas apresentaram-se mais sensíveis e desconfiadas nas relações interpessoais, assim como mais insatisfeitas com a própria aparência, comparativamente àquelas que não desejavam cirurgia estética, podendo tender a maior fechamento afetivo e social ${ }^{10}$.

A preocupação com a satisfação da imagem corporal da equipe de enfermagem surge pela possível relação desta com a comunicação interpessoal e o cuidado com o paciente, uma vez que, quando o profissional se sente bem com ele mesmo nesse aspecto, inevitavelmente ele exterioriza tal satisfação, por meio de gestos, expressões faciais e comportamentos nas relações interpessoais, tornando o cuidar uma experiência mais intensa e enriquecedora para ambos.

A proibição do uso de adornos, imposta pela Norma Reguladora 32 (NR 32) da Agência Nacional de Vigilância Sanitária (ANVISA) e vigente desde 11 de novembro de 2005 , refere-se a todos trabalhadores do serviço de saúde ${ }^{11}$ e foi implementada com certa resistência principalmente em relação ao uso de alianças e relógios de pulso, talvez por causa da representação social e cultural desses adornos. Na prática, a NR 32 ainda não é cumprida plenamente e há uma dificuldade visível na adesão de outros profissionais da equipe multiprofissional. Considerando esse contexto, supervisão, ações educativas e conscientização são atividades constantemente realizadas pelas instituições de saúde mesmo após mais de 10 anos da aprovação dessa norma.

Não é a finalidade deste estudo questionar a importância da NR 32.rRealmente, é uma norma reguladora necessária e eficaz para estabelecer medidas para proteger a segurança e a saúde dos trabalhadores de saúde em qualquer serviço 
de saúde, prevenindo acidentes e adoecimentos causados pelo trabalho ${ }^{11}$. Este estudo, portanto, justifica-se pela necessidade de avaliar os seus impactos no bem-estar dos Frente a isso, os objetivos deste estudo foram identificar a satisfação dos profissionais da equipe de enfermagem com a imagem corporal e avaliar se as características pessoais, profissionais e a necessidade do uso de adornos durante a jornada de trabalho interferem na satisfação com a imagem corporal.

Estudo transversal quantitativo, realizado com profissionais da equipe de enfermagem (enfermeiro, técnico e auxiliar de enfermagem) de dois hospitais públicos do interior do Estado de São Paulo.

Os critérios de inclusão foram enfermeiros, técnicos e auxiliares com idade entre 18 a 60 anos. Foram excluídos do estudo enfermeiros com função administrativa, como supervisores e coordenadores, devido ao nível hierárquico na instituição, ao uso não obrigatório de uniformes e à possibilidade de viés por tais motivos na satisfação com a imagem corporal.

Foi estimado um valor médio referente à população de interesse para este estudo, por meio do site do Laboratório de Epidemiologia e Estatística do Instituto Dante Pazzanese de Cardiologia. Considerando o escore médio $(81,6)$ e o desvio padrão $( \pm 14,1)$ da Escala de Avaliação da Satisfação com a Imagem Corporal ${ }^{12}$ de um estudo prévio, o Erro Máximo de Estimativa (EME) correspondente a $15 \%$ do desvio padrão $(E M E=2,115)$ e fixando o nível de significância em 5\%, o tamanho amostral estimado para aplicação deste instrumento foi de 171.

A coleta de dados ocorreu em dois momentos diferentes: de janeiro a fevereiro de 2014, e de janeiro a junho de 2015. Os instrumentos foram entregues aos participantes no ambiente de trabalho e recolhidos posteriormente, ainda no mesmo dia, após duas horas.

Foram utilizados dois instrumentos na coleta de dados: um questionário para caracterização dos participantes e a Escala de Avaliação da Satisfação com a Imagem Corporal³.

O questionário de caracterização dos participantes continha as seguintes variáveis independentes: idade, sexo, estado civil, tempo de formação, maior titulação, unidade de atuação, cargo ocupado na instituição, período de trabalho e necessidade de adornos.

A Escala de Avaliação da Satisfação com a Imagem Corporal, variável dependente do estudo, é composta por 25 itens respondidos com uma escala do tipo Likert de cinco pontos, a saber: (1) discordo totalmente; (2) discordo; (3) não concordo, nem discordo; (4) concordo; e (5) concordo totalmente. A escala é dividida em dois fatores: Satisfação com a Aparência e Preocupação com o Peso ${ }^{3}$.

O fator Satisfação com a Aparência é composto por 18 itens e define o grau de satisfação com a própria aparência, tanto no que diz respeito às características intrínsecas à própria imagem corporal quanto no que se refere à repercussão dessa imagem no ambiente externo, seja por meio de fotografias ou no outro. $O$ fator Preocupação com o Peso é composto por 7 itens e concentra itens referentes à necessidade de regulação e controle do peso como forma de se manter ou se obter uma autoimagem ideal ${ }^{3}$.

O escore total é obtido por meio da soma das pontuações obtidas nas duas subescalas e, assim, quanto maior o resultado, maior a satisfação com a imagem corporal. Os itens negativos (cargas fatoriais negativas) foram recodificados antes do cálculo do escore total em cada subescala?

A Escala de Avaliação da Satisfação com a Imagem Corporal apresenta a seguinte classificação: de 25 a 37 pontos, para totalmente insatisfeito com a imagem corporal; de 38 a 63, para insatisfeito a maioria das vezes com a imagem corporal; de 64 a 88 , para satisfeito às vezes sim, às vezes não com a imagem corporal; de 89 a 113, para satisfeito a maioria das vezes com a imagem corporal; e 114 a 125 pontos, para totalmente satisfeito com a imagem corporal 3 .

Adotou-se uma técnica de imput no banco de dados, em que dados faltantes (missing data) foram substituídos pela moda de cada afirmativa da escala. Participantes com mais de $20 \%$ de dados não respondidos foram excluídos da pesquisa.

Foram realizadas análise descritiva (média, desvio padrão e mediana) e comparativa por meio de testes estatísticos com o software Statistical Package for the Social Sciences (SPSS), versão 20.0. Para a comparação das variáveis numéricas com os escores do instrumento, foi utilizado o teste de correlação de Spearman e, na comparação das variáveis categóricas com os escores, foram utilizados o teste de Kruskal-Wallis e o teste de Mann-Whitney. A probabilidade de erro adotada nos testes foi de $p<0,05$.

0 desenvolvimento do estudo atendeu às exigências da Resolução 466/2012, vigente no país sobre ética em pesquisas envolvendo seres humanos, sendo aprovado sob número de parecer 459.959.

\section{RESULTADOS}

A amostra do estudo foi de 182 profissionais, com idade média de 32,74 anos $( \pm 8,3)$. A maioria era do sexo feminino, casada ou convivente, possuía nível técnico, ocupava o cargo de auxiliar de enfermagem e trabalhava no período diurno. Em maior frequência, estes profissionais atuavam em unidades de interação, como verifica-se na tabela 1.

A maioria sentiu falta de adornos durante a jornada de trabalho, e os adornos que os participantes mais sentiram falta foram brincos, aliança e relógio de pulso, como mostra a figura 1.

Na descrição dos adornos mencionados pelos participantes e demonstrados na figura 1 , os participantes poderiam mencionar mais de um item, portanto o total de adornos mencionados foi de 207 (100\%). São Paulo 2014-2015. 
Tabela 1. Descrição das características estudadas. São Paulo, 2014 2015.

\begin{tabular}{|c|c|c|}
\hline Características & $\mathbf{n}$ & $\%$ \\
\hline \multicolumn{3}{|l|}{ Sexo } \\
\hline Feminino & 168 & 92,3 \\
\hline Masculino & 14 & 7,7 \\
\hline \multicolumn{3}{|l|}{ Estado civil } \\
\hline Solteiro & 71 & 39,0 \\
\hline Casado ou convivente & 97 & 53,3 \\
\hline Separado, divorciado ou viúvo & 14 & 7,7 \\
\hline \multicolumn{3}{|l|}{ Titulação } \\
\hline Técnico & 132 & 72,5 \\
\hline Graduação & 24 & 13,2 \\
\hline Pós-graduação & 26 & 14,3 \\
\hline \multicolumn{3}{|l|}{ Instituiç̧ão } \\
\hline Hospital 1 & 101 & 55,5 \\
\hline Hospital 2 & 81 & 44,5 \\
\hline \multicolumn{3}{|l|}{ Cargo na instituição } \\
\hline Enfermeiro & 42 & 23,1 \\
\hline Técnico em enfermagem & 42 & 23,1 \\
\hline Auxiliar de enfermagem & 98 & 53,8 \\
\hline \multicolumn{3}{|l|}{ Unidade de atuação } \\
\hline Unidade de internação & 89 & 48,9 \\
\hline Intensiva ou semi-intensiva & 55 & 30,2 \\
\hline Pronto-socorro & 17 & 9,3 \\
\hline Centro cirúrgico & 21 & 11,5 \\
\hline \multicolumn{3}{|l|}{ Período de trabalho } \\
\hline Diurno & 137 & 75,3 \\
\hline Noturno & 45 & 24,7 \\
\hline \multicolumn{3}{|l|}{ Sente falta de adornos } \\
\hline Sim & 140 & 76,9 \\
\hline Não & 42 & 23,1 \\
\hline Total & 182 & 100 \\
\hline
\end{tabular}

Fonte: elaboração própria.

\section{Adornos mencionados pelos participantes}

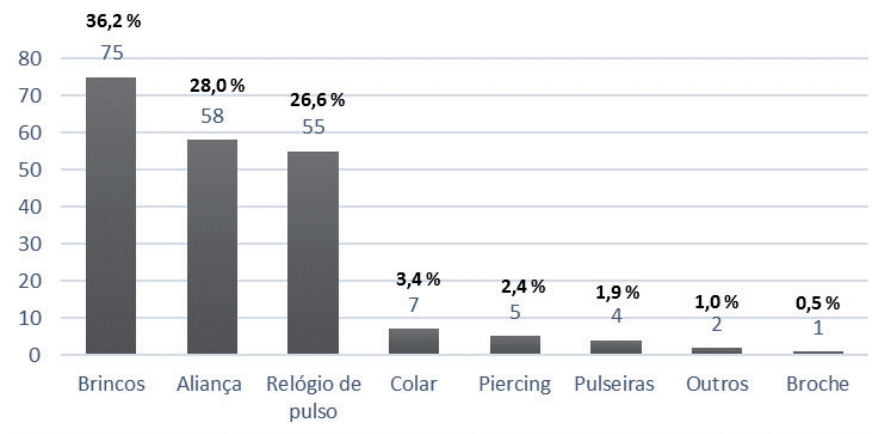

Figura 1- Adornos mencionados pelos participantes Fonte: elaboração própria.
Quanto à Escala de Avaliação da Satisfação com a Imagem Corporal, foram encontrados 56 dados faltantes, sendo os itens 24 e 25 os que apresentaram maior número de dados faltantes $(n=7)$, indicando que a satisfação do participante com sua aparência sem roupa ou a maneira que as roupas caem podiam ser questões constrangedoras, e que os participantes preferiram não responder.

0 escore médio dos participantes no fator Satisfação com a Aparência foi 61,5 ( $\pm 11,1)$, no fator Preocupação com o Peso foi $22,3( \pm 5,7)$ e no total foi de $83,8( \pm 14,1)$. A maioria dos profissionais de enfermagem estava satisfeita "às vezes sim, às vezes não" com a imagem corporal, como demonstrado na tabela 2.

Tabela 2. Descrição do escore total da Escala de Avaliação da Satisfação com a Imagem Corporal em uma classificação categórica. São Paulo, 2014-2015.

\begin{tabular}{l|c|c|c}
\hline \multicolumn{1}{c|}{ Classificação em intervalos } & $\begin{array}{c}\text { Variação da } \\
\text { pontuação }\end{array}$ & $\mathbf{n}$ & $\mathbf{\%}$ \\
\hline Totalmente insatisfeito & $25-37$ & 0 & 0 \\
\hline Insatisfeito a maioria das vezes & $38-63$ & 14 & 7,7 \\
\hline $\begin{array}{l}\text { Satisfeito às vezes sim, às } \\
\text { vezes não }\end{array}$ & $64-88$ & 106 & 58,2 \\
\hline Satisfeito a maioria das vezes & $89-113$ & 58 & 31,9 \\
\hline Totalmente satisfeito & $114-125$ & 4 & 2,2 \\
\hline Total & $25-125$ & 182 & 100 \\
\hline
\end{tabular}

Fonte: elaboração própria.

A análise descritiva dos itens da escala representou exatamente as respostas dos participantes sem a recodificação necessária para o cálculo do escore total. Observou-se, no fator Satisfação com a Aparência, mais frequência na resposta "concordo" nos itens 2 ( $n=78 ; 42,9 \%)$, $4(n=75 ; 41,2 \%), 10(n=97 ; 53,3 \%)$ e $11(n=85 ; 46,7 \%)$, e de "discordo totalmente" nos itens $8(n=78 ; 42,9 \%)$ e 13 $(n=80 ; 44 \%)$. Os participantes concordavam que tinham uma aparência tão boa quanto à da maioria das pessoas, gostavam do que viam quando se olhavam no espelho, que as outras pessoas achavam que eles tinham uma boa aparência e que se sentiam felizes com sua aparência; que discordavam totalmente sobre ter uma aparência semelhante à de outras pessoas; e que discordavam sobre sentir vergonha de sua aparência.

No fator Preocupação com o Peso, a maior frequência foi apresentada no item 22 ( $n=87 ; 47,8 \%$ ), em que os participantes discordaram totalmente sobre tentarem perder peso fazendo dietas radicais.

$\mathrm{Na}$ associação das variáveis categóricas das características dos participantes com os fatores da Escala de Avaliação da Satisfação com a Imagem Corporal, houve diferença estatisticamente significativa na comparação do fator Preocupação com o Peso com as variáveis sexo $(p=0,03)$ e estado civil $(p=0,02)$, mostrando que a preocupação com o peso foi maior entre participantes do sexo masculino e solteiros, verficada na tabela 3 . 
Tabela 3. Comparação das características da amostra com o escore total e os domínios da Escala de Avaliação da Satisfação com a Imagem Corporal. São Paulo, 2014-2015.

\begin{tabular}{|c|c|c|c|c|c|c|}
\hline & \multicolumn{3}{|c|}{ Satisfação com a aparência } & \multicolumn{3}{|c|}{ Preocupação com o peso } \\
\hline & Média & DP & Valor de $\mathrm{p}$ & Média & DP & Valor de $p$ \\
\hline Gênero* & & & 0,46 & & & 0,03 \\
\hline Feminino & 61,31 & 10,83 & & 22,03 & 5,70 & \\
\hline Masculino & 64,14 & 13,63 & & 25,50 & 5,06 & \\
\hline Estado civil† & & & 0,25 & & & 0,02 \\
\hline Solteiro & 62,92 & 10,41 & & 23,61 & 5,32 & \\
\hline Casado ou convivente & 60,81 & 11,64 & & 21,72 & 5,74 & \\
\hline Separado, divorciado ou viúvo & 59,35 & 9,73 & & 19,64 & 6,34 & \\
\hline
\end{tabular}

*Teste de Mann-Whitney; †teste de Kruskal-Wallis. DP: desvio padrão. Fonte: elaboração própria.

As associações entre os fatores e as variáveis titulação, cargo na instituição, unidade de atuação, período de trabalho e sensação de falta de adornos, bem como a correlação entre os fatores com a variável numérica idade, não obtiveram diferenças estatisticamente significativas.

\section{DISCUSSÃO}

Neste estudo, os profissionais da equipe de enfermagem apresentaram moderada satisfação com a imagem corporal e coerência nas respostas. Os resultados deste estudo corroboram, em parte, resultados de uma pesquisa que avaliou a satisfação de 700 universitários da área da saúde também com a aplicação da Escala de Avaliação da Satisfação com a Imagem Corporal ${ }^{5}$. Ao avaliarem a própria aparência, $41,7 \%$ estavam satisfeitos e $31,1 \%$ extremamente satisfeitos com a aparência. Porém, os autores encontram contradições: ao mesmo tempo que os estudantes não gostam de seu físico, também acham que tem um corpo bom; ao mesmo tempo em que estão felizes com a aparência, eles mudariam muitas coisas nela; e ao mesmo tempo em que tentam mudar o peso, também estão satisfeitos com ele. Embora em ambos os trabalhos a satisfação com a imagem corporal tenha sido moderada, não foram encontradas contradições evidentes no presente estudo.

A preocupação com o peso está intimamente ligada à satisfação da imagem corporal. Neste estudo, o excesso de peso foi um problema e relacionou-se aos resultados encontrados por outros autores ${ }^{8 \otimes 13 \$ 14}$. Em um estudo, foi verificado que a preocupação com o peso pode ser algo distorcido e influenciar negativamente na saúde das pessoas ${ }^{8}$,e que o excesso de peso é mais elevado em pessoas mais velhas, com menor titulação e que trabalhavam mais horas por dia $^{13}$. Esteve, ainda, associado com uma redução da qualidade de vida ${ }^{14}$, gerando percepções negativas ${ }^{15}$. Já a satisfação com o peso é fortemente influenciada por ideais de magreza divulgados pela mídia ${ }^{5}$.

Na comparação com a imagem corporal e a satisfação com o peso, um estudo encontrou que, entre alunas que apresentavam níveis de preocupação com a imagem corporal normal/leve, $58,7 \%$ gostaria de perder $2 \mathrm{~kg}$ ou mais, e, naquelas com alteração moderada/grave da autoimagem corporal esse mesmo desejo atingiu quase a totalidade das universitárias (94,3\%), o que corresponde a um alto grau de distorção detectado e a um fator de risco para transtornos na esfera da alimentação8.

Em um estudo realizado com 2.103 enfermeiras americanas com idade média de 46 anos, em que a maioria trabalhava em hospitais, foi avaliada a relação entre estresse no trabalho, turnos de trabalho e obesidade. Os resultados mostraram que $55 \%$ das participantes estavam acima do peso ou obesas. Não foi encontrada nenhuma relação com o sobrepeso ou obesidade e o local de trabalho. Entretanto, quando foram comparadas enfermeiras abaixo do peso ou com peso normal com as que estavam acima do peso ou obesas, estas eram, em média, 2,5 anos mais velhas, possuíam menor titulação, eram mais experientes, trabalhavam mais horas por dia e relatavam que possuíam mais problemas para dormir e de saúde do que as demais ${ }^{13}$.

0 efeito do excesso de peso, a obesidade e a qualidade de vida e satisfação com a imagem corporal foram condições estudadas entre habitantes da Lituânia. A pesquisa incluiu medições físicas, informações sobre fatores de risco relacionados ao estilo de vida e o questionário de qualidade de vida da Organização Mundial da Saúde. Os resultados mostraram que $73 \%$ de homens e mulheres tinham excesso de peso. Entre as mulheres, o excesso de peso corporal foi associado a uma redução da qualidade de vida nos domínios psicológico e de independência. Os homens com excesso de peso relataram melhor qualidade de vida global. Pessoas obesas estavam menos satisfeitas com sua imagem corporal, em comparação com aquelas com peso normal ${ }^{14}$.

Estudo que investigou as atitudes de 352 estudantes de enfermagem e 198 enfermeiros, em relação às pessoas obesas, por meio de duas escalas (Fat Phobia Scale e Attitudes Toward Obese Adult Persons Scale), encontrou que ambos, enfermeiros e estudantes, têm percepções 
negativas da obesidade e não são suscetíveis a atribuírem características positivas para os indivíduos obesos ${ }^{15}$.

Estudo que analisou a imagem corporal de adultos fortemente influenciada por ideais de magreza identificou que a satisfação com a imagem corporal não se mostrou associada ao sexo e à idade, mas foi influenciada pelo tamanho corporal real. Os indivíduos de peso normal e abaixo do peso apresentaram níveis de satisfação corporais maiores que os dos grupos de sobrepeso e acima do peso ${ }^{5}$.

Em estudo anterior ${ }^{12}$, os profissionais mais seguros e satisfeitos com a aparência não sentiram falta de adornos. Ao relacionar a sensação de falta de adornos com a satisfação da imagem corporal e o autoconceito profissional de 46 membros da equipe de enfermagem, indivíduos mais realizados, mais autoconfiantes, que mais percebem a influência do trabalho na saúde e mais satisfeitos com a aparência não sentiram falta de adornos no ambiente hospitalar. Apesar de o uso de adornos ser uma comunicação não verbal importante, a proibição não incomodou as pessoas mais satisfeitas com a própria aparência. Entretanto, o inverso também é verdadeiro: profissionais menos satisfeitos com a aparência física sentiram falta de adornos, por isso, esse assunto é algo atual e que precisa ser mais estudado.

A escolha de adornos e acessórios é orientada por grupo, status, relações e função do indivíduo na sociedade. Assim, adornos também são uma forma de comunicação não verbal das intenções e dos sentimentos das pessoas nos diferentes contextos ${ }^{4}$. A NR 32 considera adornos: alianças, anéis, pulseiras, relógios de uso pessoal, colares, brincos, broches, piercings expostos, gravatas e crachás pendurados com cordão ${ }^{11}$. Existem adornos com importantes significados sociais. As mãos são, talvez, a parte mais ativa no corpo humano e são embelezadas com anéis porque são muito visíveis e móveis durante os encontros sociais. Além desse papel embelezador, o uso da aliança nas mãos, por exemplo, representa o estado matrimonial do indivíduo para a sociedade; o fato de ser utilizada no quarto dedo também tem um sentido cultural, esse dedo possui menor mobilidade que os outros, portanto é o local apropriado para colocar um símbolo de limitação da independência ${ }^{4-16}$.

Nesta pesquisa, a preocupação com o peso foi maior nos indivíduos solteiros e do sexo masculino. Provavelmente, o instinto de atratividade física tenha sido o motivo de os solteiros se preocuparem mais com peso corporal neste estudo. Quanto ao achado em relação ao sexo, talvez o motivo tenha sido a ênfase no contexto histórico atual para a saúde e o controle do peso, bem como o aumento no consumo de produtos de beleza pelos homens contemporâneos.

A relação entre a masculinidade e o consumo de beleza foi foco de pesquisa qualitativa usando técnica projetiva. Foram entrevistados 10 jovens homens de classe econômica alta no Rio de Janeiro, e realizados três exercícios de projeção. No primeiro, foram apresentadas quatro imagens de modelos femininas; os entrevistados foram questionados sobre a beleza delas, apontaram a mais bonita e tentaram descrever suas práticas de beleza. No segundo exercício de imaginação, os entrevistados deveriam se colocar na posição de uma mulher e, assim, escolher, dentre quatro fotos de modelos masculinos, o homem mais atraente e o menos atraente, explicando tais escolhas. 0 último exercício foi uma autoavaliação em relação à beleza. Os resultados mostraram que os entrevistados estavam bem à vontade para comentar sobre as fotos femininas. No entanto, sobre a beleza dos modelos masculinos, os comentários eram feitos na terceira pessoa, e os entrevistados mostraram resistência em responder às perguntas. A fase de autoavaliação mostrou-se bastante difícil para os participantes. A comparação de sua aparência física com a dos modelos masculinos pareceu gerar desconforto: o contraste entre as fotos de corpos e rostos "perfeitos" e suas próprias imperfeições pareceu suscitar - ou reavivar - insatisfações, inseguranças e questões pessoais, talvez íntimas demais para serem verbalizadas ${ }^{17}$.

\section{CONCLUSÃO}

Em geral, a equipe de enfermagem referiu estar moderadamente satisfeita com a imagem corporal. A preocupação com o peso foi influenciada pelo sexo e estado civil, sendo maior nos homens e solteiros. A associação entre os fatores da Escala de Avaliação da Satisfação com a Imagem Corporal e sensação de falta de adornos não foi conclusiva neste estudo, entretanto a maioria dos profissionais sentiram falta deles e os mais mencionados foram brincos, aliança e relógio de pulso.

Este estudo alerta sobre a importância do bem-estar do profissional consigo mesmo e no ambiente de trabalho em relação a sua imagem corporal e que esses aspectos devem ser estimulados pelas instituições de saúde e gestores, principalmente pelas possíveis relações com o cuidar: o cuidar do outro não pode estar desvinculado do cuidar de si.

\section{REFERÊNCIAS}

1. Kakeshita IS, Almeida SS. Relação entre índice de massa corporal e a percepção da auto-imagem em universitários. Rev Saúde Pública [Internet]. 2006;40(3):497-504. Disponível em: http://www.scielo.br/pdf/rsp/v40n3/19.pdf

2. Silva GA, Lange ES. Imagem corporal: a percepção do conceito em indivíduos obesos do sexo feminino. Psicologia Argumento [Internet]. 2010;60(28):43-54. Disponível em: http://www2.pucpr.br/reol/pb/index.php/ pa?dd1=3509\&dd99=view \&dd98=pb.

3. Ferreira MC, Leite NG. Adaptação e validação de um instrumento de avaliação da satisfação com a imagem corporal. Avaliação Psicológica [Internet]. 2002;2(1):1419. Disponível em: http://pepsic.bvsalud.org/scielo. php?script=sci_arttext \&pid=S1677-04712002000200007.

4. Silva MP. Comunicação tem remédio: a comunicação nas relações interpessoais de saúde. $8^{a}$ ed. São Paulo: Loyola; 2013. 
5. Saur AM, Pasian SR. Satisfação com a imagem corporal em adultos de diferentes pesos corporais. Avaliação Psicológica [Internet]. 2008;7(2):199-209. Disponível em: http:// repository.usp.br/single.php?_id=001725824.

6. Sobreira CD. Relação entre percepção auto-referida da imagem corporal de estudantes universitários da área de saúde e desenvolvimento da identidade corporal: um olhar psicodramático. DPSedes - Departamento de Psicodrama Instituto Sedes Sapientiae. 2008.

7. Schwartz MB, Brownell KD. Obesity and body image. Body Image [Internet]. 2004;1(1):43-56. Disponível em: https:// www.ncbi.nlm.nih.gov/pubmed/18089140.

8. Bosi MLM, Luiz RR, Morgado CMC, Costa MLS, Carvalho RJ. Autopercepção da imagem corporal entre estudantes de nutrição: um estudo no município do Rio de Janeiro. J Bras Psiquiatr [Internet]. 2006;55(2):108-13. Disponível em: http://www.scielo.br/pdf/jbpsiq/v55n2/v55n2a03.

9. Bracht DM, Piasetzki CT, Busnello MB, Berlezi EM, Franz LB, Boff ET. Percepção da autoimagem corporal, estado nutricional e prática de atividades físicas de universitários do Rio Grande do Sul. Mundo Saúde [Internet]. 2013;37(3):34353. Disponível em: http://bvsms.saude.gov.br/bvs/artigos/ mundo_saude/percepcao_autoimagem_corporal_estado_ nutricional.pdf.

10. Sante AB, Pasian SR. Imagem corporal e características de personalidade de mulheres solicitantes de cirurgia plástica estética. Psicologia: Reflexão e Crítica. [Internet] 2011;24(3):421-9.Disponível em:http://www.scielo.br/scielo. php?script=sci_arttext \& pid=S0102-79722011000300003.

11. Brasil. Ministério do Trabalho e Emprego. Norma Regulamentadora no. 32. Segurança e Saúde no Trabalho em Estabelecimentos de Saúde. Brasília, DF: Ministério do Trabalho e Emprego; 2005.

12. Puggina AC, Cavalheiro AC, Trentino JP, Castro P, Silva MJ. Relação entre necessidade de adornos com satisfação com imagem corporal e autoconceito profissional da equipe de enfermagem. Rev Anna Nery [Internet]. 2015;19(4):563-70. Disponível em: http://www.scielo.br/pdf/ean/v19n4/14148145-ean-19-04-0563.pdf.

13. .Han K, Trinkoff AM, Storr CL, Brown JG. Job stress and work schedules in relation to nurse obesity. J Nurs Adm [Internet]. 2011;40(11):488-95. Disponível em: https://www.ncbi.nlm. nih.gov/pubmed/22033319.

14. Bacevičienè M, Rèklaitienè R, Tamošiūnas A. Effect of excess body weight on quality of life and satisfaction with body image among middle-aged Lithuanian inhabitants of Kaunas city. Medicina (Kaunas) [ Internet]. 2009;45(7):565-73. Disponível em: https://www.ncbi.nlm.nih.gov/pubmed/19667752.

15. Poon MY, Tarrant M. Obesity: attitudes of undergraduate student nurses and registered nurses Obesity: attitudes of undergraduate student nurses and registered nurses. J Clin Nurs [Internet]. 2009;18:2355-65. Disponível em: https:// www.ncbi.nlm.nih.gov/pubmed/19374692.

16. Fontes AO, Borelli FC, Casotti LM. Como ser homem e ser belo? Um estudo exploratório sobre a relação entre masculinidade e o consumo de beleza. Rev Eletrôn Adm REAd [Internet]. 2012;72(2):400-32. Disponível em: http://seer.ufrgs.br/read/ article/view/38396. 